\title{
Effects of Thymoquinone on Tramadol Induced Toxicity in Epididymis of Adult Male Rabbits (Histological \& Immunohistochemical Study)
}

\author{
Ayman Mohammad Mousa ${ }^{1,2}$ \\ ${ }^{1}$ Department of Basic Health Sciences, CAMS, Qassim University, Buraydah, Kingdom of Saudi Arabia \\ ${ }^{2}$ Department of Histology and Cell Biology, Faculty of Medicine, Benha University, Benha, Egypt
}

\section{Email address:}

a.mousa@qu.edu.sa

\section{To cite this article:}

Ayman Mohammad Mousa. Effects of Thymoquinone on Tramadol Induced Toxicity in Epididymis of Adult Male Rabbits (Histological \& Immunohistochemical Study). International Journal of Clinical and Developmental Anatomy. Vol. 5, No. 1, 2019, pp. 1-7. doi: 10.11648/j.ijcda.20190501.11

Received: February 19, 2019; Accepted: March 8, 2019; Published: April 2, 2019

\begin{abstract}
Millions of patients who administer or abuse tramadol to relieve chronic pain may suffer from infertility. Thymoquinone (TQ) is a major active component of nigella sativa oil, which has antioxidant and anti-inflammatory effects. The present work aimed to clarify effects of tramadol and TQ on epididymis of adult male rabbits. Forty adult male rabbits aged 3 months were used for one month \& divided equally into 4 groups: Group 1 as a control group; group 2 was treated with tramadol $30 \mathrm{mg} / \mathrm{kg}$ orally/day, group 3 was treated with TQ $20 \mathrm{mg} / \mathrm{kg}$ orally/day and group 4 as a protected group that was treated with TQ $20 \mathrm{mg} / \mathrm{kg} \&$ tramadol $30 \mathrm{mg} / \mathrm{kg}$ orally/day. The epididymis was processed for histological and immunohistochemical (IHC) study for detection of epididymal structure and caspase-3 as an indicator of epididymal apoptotic changes. Statistical analysis of apoptotic index, area\% of collagen and caspase-3 immunoexpression was carried out. Group 2 showed epithelial thinning of epididymal wall, degeneration and vacuolation of epithelial cells with disturbance of stromal collagen fibers. Also, a significant increase of apoptotic index, area $\%$ of collagen and caspase-3 cytoplasmic immunoexpression in epididymal epithelium was noticed in group 2. While, Group 3 and 4 showed improvement of the histological and IHC changes described in group 2. It is concluded that tramadol intake exerted toxic effects on epididymal structure while, TQ minimized these effects. Therefore, intake of TQ with tramadol is advised to protect the epididymis from the hazardous effects of tramadol abuse.
\end{abstract}

Keywords: Tramadol, Thymoquinone, Apoptosis, Epididymis, Male Fertility, Immunohistochemistry

\section{Introduction}

There is a growing interest in male reproductive toxicity due to hazardous effects of drugs, industrial chemicals and environmental pollutions [1]. Epididymal toxicity gains importance as it led to male infertility where epididymis improves maturation, post-testicular transport and storage of spermatozoa. However, more information about the effects of tramadol on epididymal oxidative stress is required [2].

The epididymis is a highly convoluted single long duct that links the efferent ducts to vas deferens and extends from the caput to cauda epididymis which are lined with pseudostratified columnar epithelium (PSCE) that is formed of principal, apical and basal cells. The first two segments are considered areas of sperm maturation within its lumen, while the last portion represents a storage region [3].

The basal cells (BCs) and apical cells (ACs) are intermingled between numerous principal cells (PCs). The tall columnar PCs are very active in protein synthesis, secretion and transport of tiny molecules with absorption of fluid and particulate matter. While, the small elongated BCs play an active role in detoxification with numerous apical vesicles and lysosomes that remove materials actively from the lumen [4]. The main function of epididymis is the production of a specific microenvironment to mature and transport sperms through absorption and secretion of different protein substances. These substances help sperms to survive, induce progressive sperm motility and acquire a 
fertilizing capacity [5].

Although the common usage of tramadol among youth leads to addiction and dramatic effects on structure of reproductive organs, there is a little information about its reproductive effects especially on the epididymis [6]. Tramadol is an opioid analgesic with a chemical synthetic structure similar to codeine that stimulates dopamine receptors with analgesic effect less than morphine [7].

It inhibits the release of gamma amino butyric acid in the central nervous system (CNS) and is used to relieve acute or chronic severe pain [8]. Tramadol absorption is more rapid in the small intestine than morphine to reach its highest peak after 6 hours and is widely distributed throughout the body, especially the liver and kidneys [9]. Repeated administration of tramadol decreases its clearance and increases its toxic metabolites leading to CNS stimulation that causes headache, dizziness or constipation. [10]. In addition, tramadol toxicity is related to the overdose as people who develop tolerance need large doses to obtain the same desired effects. Tramadol overdose may cause death due to cardiopulmonary arrest, hypoglycemia or hepatic failure [11]. Therefore, it is interesting to explore if repeated tramadol administration induces histopathological and IHC alterations on the epididymis of male rabbits or no.

Over many centuries humans have been turning to nature to discover substances for treatment of multiple diseases through enhancing the antioxidant defense enzymes, as superoxide dismutase and catalase which are very important to scavenge free radical species [12]. Nigella sativa (NS) seeds are amazing natural herb that has been used against several diseases. It contains $30 \%$ fixed oil and $0.1 \%$ volatile oils where; TQ constitutes $30 \%$ of these volatile oils [13].

TQ is the major bioactive component of NS oil that has most of its biological, antioxidant, immunomodulatory and anti-inflammatory effects however, TQ effects on the histological structure of epididymis have not been investigated in depth and needs to be an active area of research nowadays [14].

This work aimed to explore the histological and IHC changes induced by prolonged administration of tramadol on the epididymis of male rabbits and the role of TQ in reducing these effects.

\section{Materials and Methods}

Forty adult male New Zealand white rabbits, aged 3 months and weighing (1.0-1.4 kg), were used in this study and housed for one month at the animal house of Qassim University, KSA in metal cages. The rabbits were feeding tap chow diet and water ad libitum at $23^{\circ} \mathrm{C}$.

\subsection{Experimental Design}

The experimental protocol and procedures of this study were accepted by the ethical scientific research committee of Qassim University, KSA. Rabbits were divided into 4 groups (10 rabbits for each group) administered doses of drugs suspended in $2 \mathrm{ml}$ of distilled water (DW) orally by a stomach tube once daily for one month as follow:

(1) Group1 (control = G1) received $2 \mathrm{ml}$ of DW only.

(2) Group 2 (tramadol treated group $=\mathrm{G} 2$ ) was administered $30 \mathrm{mg}$ of tramadol $/ \mathrm{kg}$ of BW [15].

(3) Group 3 (TQ-treated group $=\mathrm{G} 3$ ) was administered 20 $\mathrm{mg}$ of TQ $/ \mathrm{kg}$ of BW [16].

(4) Group 4 (protected group $=$ G4) was administered 20 $\mathrm{mg}$ of TQ and $30 \mathrm{mg}$ of tramadol $/ \mathrm{kg}$ of BW. Tramadol $\mathrm{HCl}$ (Tramal) was purchased as $50 \mathrm{mg}$ capsules from Mina-Pharm, Egypt and TQ was purchased from Sigma-Aldrich, USA as crystals.

\subsection{Light Microscopic and IHC Study}

By the end of experiment, all animals were sacrificed and the epididymis was incised immediately. Specimens were fixed overnight in Bouin's fluid and processed to obtain 5 um tissue sections then stained with H\&E and Masson's trichrome stains according to the standard histological protocols in Bancroft et al. to investigate the general histological structure under light microscopy [17]. Avidin Biotin peroxidase Complex (ABC) technique (LSAB 2 Kit; Dako) was used in IHC reactions and rabbit monoclonal antibody of caspase-3 protein (anti- caspase-3; Abcam, UK) was used with biotinylated antirabbit IgG1 secondary antibody (Abcam, UK) [18]. Sections kept in 3\% H2O2, incubated with rabbit monoclonal antibody of caspase-3 protein for 1 night at $4^{\circ} \mathrm{C}$, followed by incubation with biotinylated antirabbit IgG1 secondary antibody in $\mathrm{ABC}$ solution. The binding of antibody to sections was visualized by DAB reaction (Abcam, UK). Finally, these sections were stained with Mayer's hematoxylin and negative control sections had the same procedure without the primary antibodies. Positive controls were used as the manufacturer instructions of primary antibody [19].

\subsection{Morphometric Study and Statistical Analysis}

Digital photomicrographs were taken and analyzed for general histology by using an imaging morphometry system, "consisting of light microscope and digital camera (Leica Microsystems, Germany). The images were transformed into a grey image and then delineated to choose the areas exhibiting positive reactivity with accumulation of all grades of reaction" [20]. The morphometrical parameters of control and treated groups were measured in the $\mathrm{CE}$ tubules of epididymis in 10 random non overlapping fields from 5 slides of each rabbit in all groups at magnification 1000x to determine:

A The epididymal apoptotic index (AI) \% by counting the mean number of shrunken and vacuolated epithelial cells (apoptotic cells)/100 cells of epididymal CE ducts stained by H\&E.

B The area $\%$ of collagen fibers in epididymal CE tubules stained by Masson trichrome.

$\mathrm{C}$ The area \% of caspase-3 immunoreaction in epithelial cells of CE ducts.

The computer program SPSS version 16 (Chicago, Illinois, 
USA) was used to analyze the data. All data were expressed as mean \pm standard deviation (SD). One way ANOVA test followed by post-hoc tukey was used to compare the animal intergroup comparisons and $\mathrm{P}$ value less than 0.05 was considered significant.

\section{Results}

\subsection{Light Microscopic Histological and IHC Results}

(1) Control group (G1): Histological examination of epididymis from G1 showed PSCE resting on a thin lamina propria (LP) and multiple layers of circular smooth muscle fibers (SMF) while the CE lumen (L) had spermatozoa (S). PSCE had mainly tall columnar PCs with basal nuclei, few polygonal BCs and some ACs with dark nuclei in figure1A. Masson trichrome stained sections of G1 had a thin interstitium of loose areolar connective tissue (LACT) in figure 2A while; the immunostained sections showed a weak cytoplasmic immunoexpression of caspase-3 in epithelial cells of CE ducts in figure $3 \mathrm{~A}$.

(2) Tramadol-treated group (G2): Examination of CE sections of $\mathrm{G} 2$ revealed PSCE resting on a thick LP
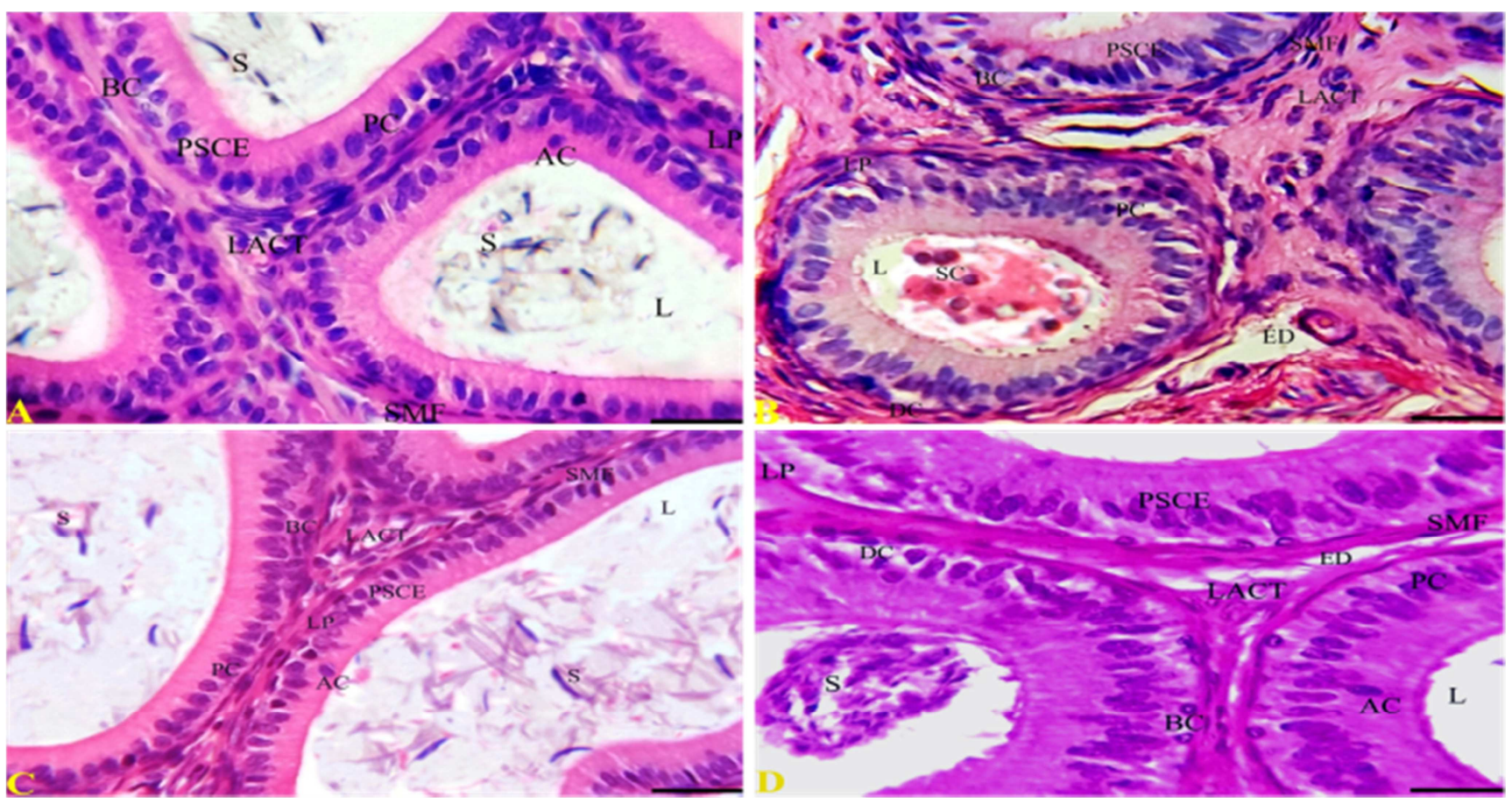

Figure 1. Photomicrographs of sections stained by H\&E from corpus epididymis (CE) ducts of: A-Control group (G1); B-Group treated with tramadol (G2); $C$ - Group treated with TQ (G3); D- Group treated with TQ and tramadol (G4).

A- Control group (G1) shows pseudostratified columnar ciliated epithelium (PSCE) which reveals principal cells (PC), basal cells (BC) and apical cells (AC). PSCE rests on a thin lamina propria (LP) and peritubular multiple circular smooth muscle fibers (SMF) while, the ducts are separated by a thin interstitium of loose areolar connective tissue (LACT) and their lumens (L) have spermatozoa (S).

B- Group treated with tramadol (G2) shows PSCE which has many shrunken, vacuolated and degenerated cells (DC) of PC and BC. PSCE rests on LP and SMF while, the ducts are separated by a thick interstitium of LACT with marked edema (ED) and their lumens (L) have some sloughed epithelial cells (SC).

C- Group treated with TQ (G3) shows PSCE that reveals PC, BC and AC. PSCE rests on a thin LP and SMF while, the ducts are separated by a thin interstitium of LACT and their lumens (L) have $\mathrm{S}$.

D- Group treated with TQ and tramadol (G4) shows apparently normal PSCE lining the duct which has PC, BC and AC that are similar to G1 however, a few DC with focal vacuoles are present. PSCE rests on a thin LP and SMF nearly similar to G1 rabbits while, the ducts are separated by a thick interstitium of LACT with minimal ED and their lumens have S. Bar= $10 \mu \mathrm{m}$. sloughed cells (SC). Many PCs and BCs were cytoplasm in figure1B. While; the interstitium showed a thick LACT with marked edema (ED) in figure 2B and the immunostained sections showed a strong cytoplasmic immunoexpression of Caspase-3 in $\mathrm{CE}$ epithelial cells in figure 3B. nearly the same histological and IHC structure of G1 rabbits in figures $1 \mathrm{C}, 2 \mathrm{C}$ and $3 \mathrm{C}$.

(4) TQ and tramadol-treated group (G4): The CE sections resting on a thin LP and multiple circular SMF. nearly similar to $\mathrm{G} 1$ cells with basal nuclei however; a few degenerated PCs were seen with focal vacuoles (DC) in figure1D. Masson trichrome stained sections of G4 had a thick interstitium of LACT with a minimal wille the immunostained sections showed a weak cytoplasmic immunoexpression of caspase-3 in epithelial cells of CE ducts in figure 3D. and SMF. The lumen of CE was narrow and had some of G4 showed apparently normal PSCE lining the duct 

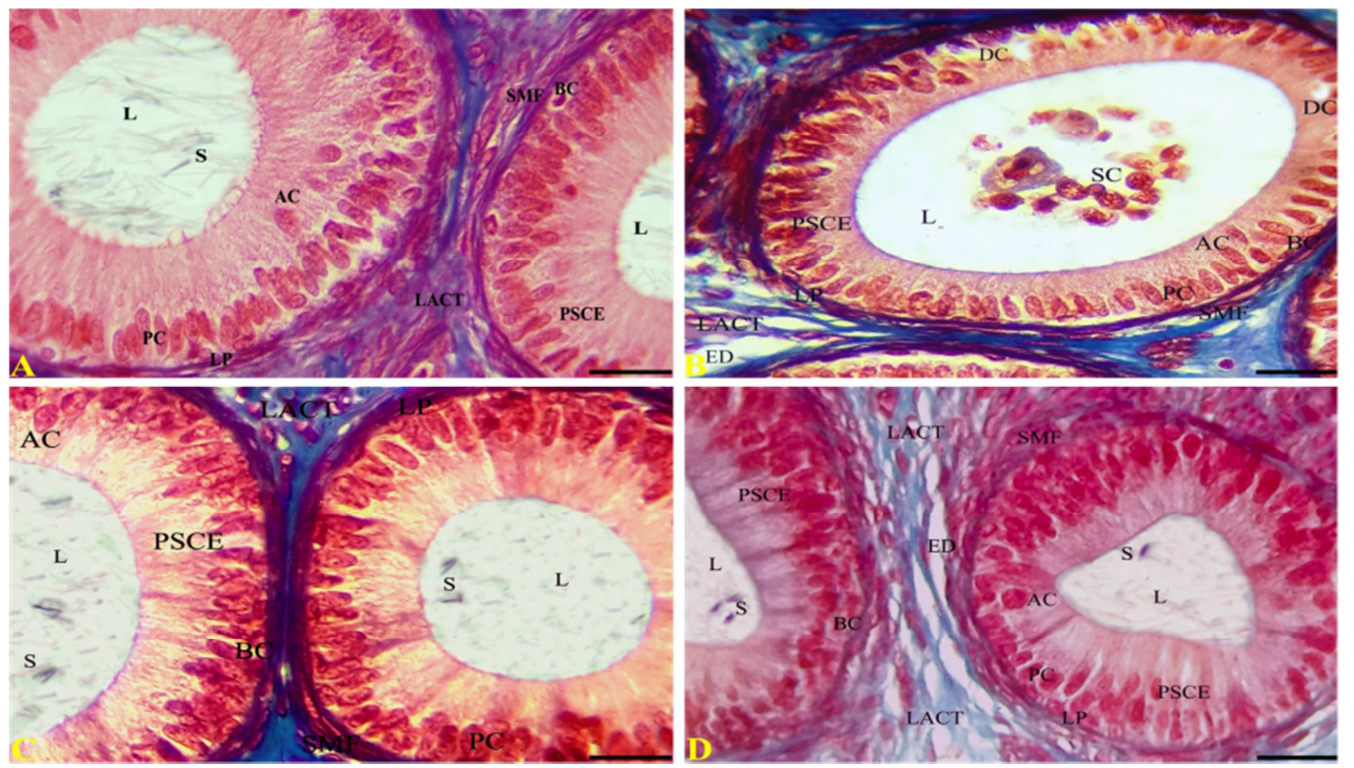

Figure 2. Photomicrographs of sections stained by Masson's trichrome from CE ducts of: A-Control group (G1); B- Group treated with tramadol (G2); $C$ - Group treated with $T Q$ (G3); D- Group treated with $T Q$ and tramadol (G4).

A- G1 shows PSCE that lines the duct and has PC, BC and AC. PSCE rests on a thin LP and peritubular SMF while the ducts are separated by a thin interstitium of LACT and their lumens (L) have S.

B- G2 shows PSCE that reveals many vacuolated DC of PC, BC and AC. PSCE rests on a thick LP and SMF while the ducts are separated by a thick interstitium of LACT with marked ED and their lumens (L) have some SC.

C- G3 shows PSCE that has normal PC, BC and AC. PSCE rests on a thin LP and SMF while the ducts are separated by a thin interstitium of LACT and their lumens (L) have $\mathrm{S}$.

D- G4 shows CE ducts that reveals apparently normal PSCE similar to G1 rabbits. PSCE has nearly normal PC, BC and AC while rests on a thin LP and SMF. The ducts are separated by a thick interstitium of LACT with minimal ED and their lumens (L) have S. Bar $=10 \mu \mathrm{m}$.
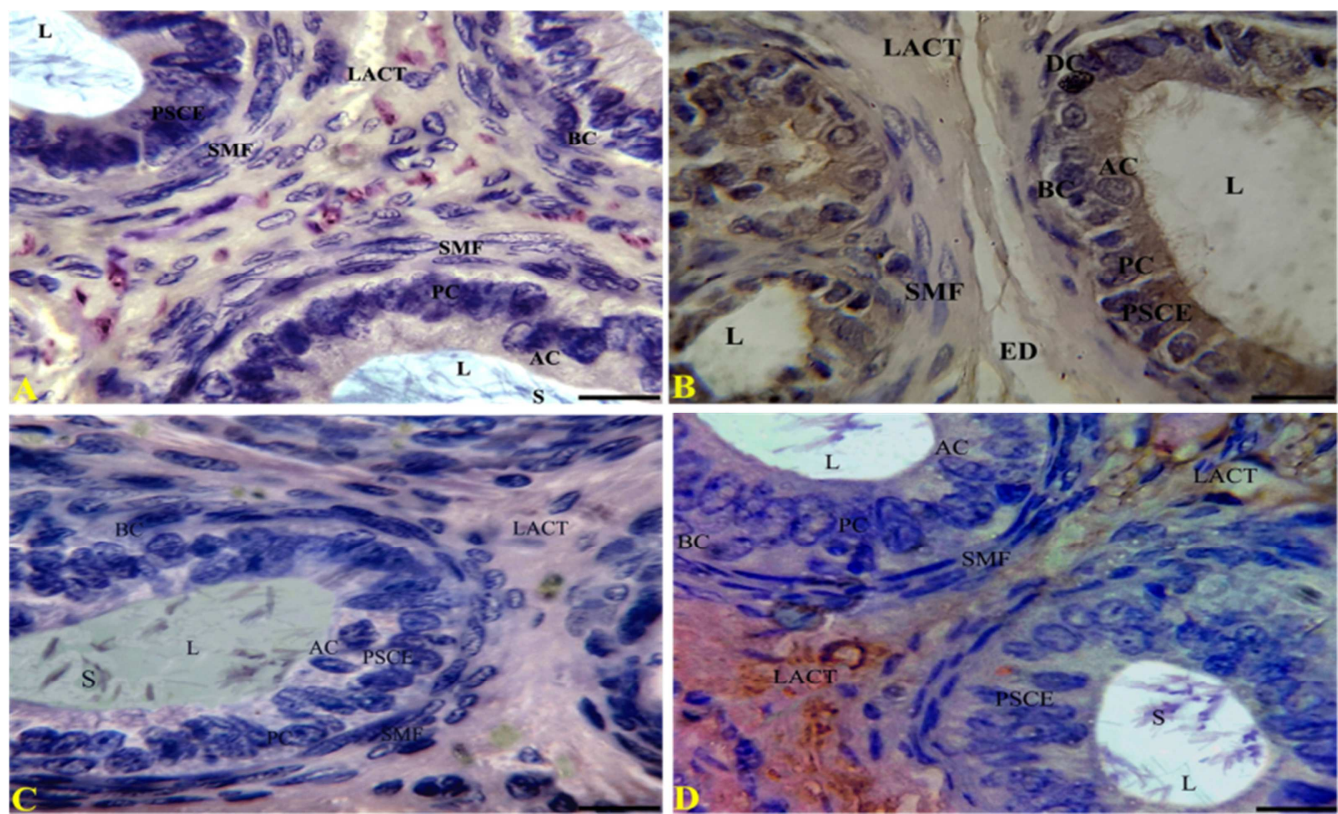

Figure 3. Photomicrographs of caspase-3 immunostained sections from CE ducts of: A-Control group (G1); B- Group treated with tramadol (G2); $C$ - Group treated with TQ (G3); D- Group treated with $T Q$ and tramadol (G4).

A- G1 shows a weak immunoexpression of Caspase-3 in all epithelial cells of PSCE (PC, BC and AC). PSCE rests on a peritubular SMF while the ducts are separated by a thin interstitium of LACT and their lumens (L) have some $\mathrm{S}$.

B- G2 shows a strong immunoexpression of caspase-3 in epithelial cells of PSCE (PC, BC and AC) and reveals some vacuolated DC. PSCE rests on a peritubular SMF while the ducts are separated by a thick interstitium of LACT with marked ED and their lumens (L) have no S.

C- G3 shows a weak immunoexpression of caspase-3 in epithelial cells of PSCE (PC, BC and AC). PSCE rests on a peritubular SMF while the ducts are separated by a thin interstitium of LACT and their lumens (L) have S.

D- G4 shows a weak immunoexpression of caspase-3 in epithelial cells of PSCE (PC, BC and AC). PSCE rests on a peritubular SMF while the ducts are separated by a thick interstitium of LACT and their lumens (L) have S. Bar $=10 \mu \mathrm{m}$. 


\subsection{Morphometric Results and Statistical Analysis}

The epididymal apoptotic index $\%$ of epithelial cells, area $\%$ of collagen fibers in epididymal wall and area $\%$ of Caspase-3 immuno-expression in epithelial cells increased significantly in G2 compared to GI, G3 and G4 as presented in table 1.

Table 1. Shows the apoptotic index \% of epithelial cells, area \% of collagen fibers and area \% of Caspase-3 immuno-expression in all groups of rabbits. The data are presented as the mean values $\pm S D$.

\begin{tabular}{|c|c|c|c|c|}
\hline Parameter & Mean \pm SD of G1 & Mean \pm SD of G2 & Mean \pm SD of G3 & Mean \pm SD of G4 \\
\hline Apoptotic index $\%$ of epithelial cells & $3 \pm 1$ & $19 \pm 4$ & $2 \pm 1$ & $7 \pm 2$ \\
\hline$P$ value & - & $0.02 * *$ & $0.07 *$ & $0.28^{*}$ \\
\hline Area $\%$ of collagen fibers in epididymal wall & $5 \pm 0.61$ & $9 \pm 1.93$ & $4 \pm 0.71$ & $8 \pm 0.95$ \\
\hline P value & - & $0.01 * *$ & $0.09 *$ & $0.27 *$ \\
\hline Area $\%$ of Caspase- 3 immuno-expression in epithelial cells & $2 \pm 0.77$ & $14 \pm 2.39$ & $3 \pm 1.13$ & $5 \pm 1.85$ \\
\hline$P$ value & - & $0.01 * *$ & $0.08 *$ & $0.23^{*}$ \\
\hline
\end{tabular}

$\mathrm{SD}=$ Standard deviation, Non-significant* and Significant** when P value is less than 0.05 as G II, III and IV compared with GI.

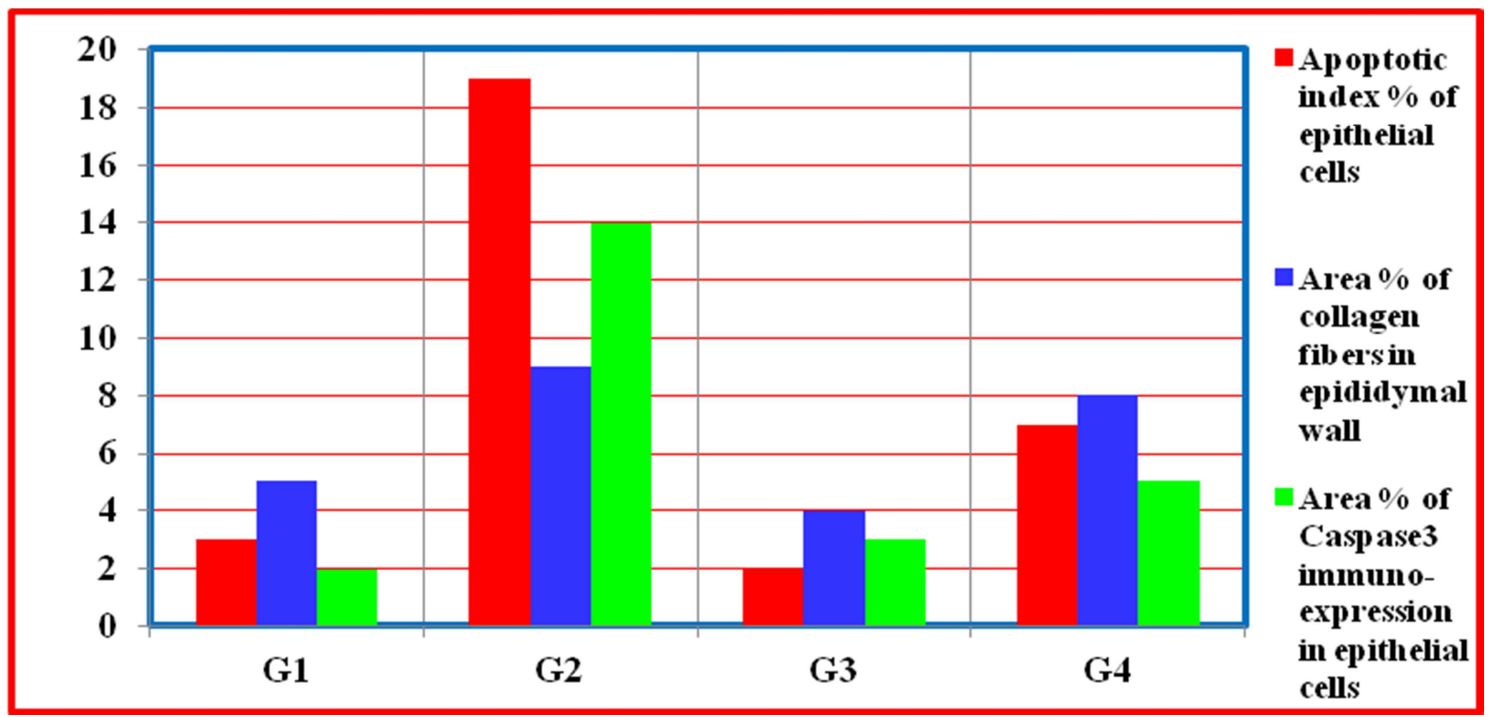

Figure 4. Shows the apoptotic index \% of epithelial cells, area \% of collagen fibers and area \% of caspase-3 immuno-expression in all groups of rabbits.

\section{Discussion}

The epididymis has a vital role for transport, functional maturation to gain motility, capacitation to fertilize oocytes and storage of mammalian sperms during epididymal transit while, many evidences determined the hazardous effects of drugs on male reproduction so; tramadol toxicity should be considered during its therapy with large doses for prolonged periods [21]. The present work examined the effects of tramadol on epididymis of adult male rabbits and the protective effects of TQ on these alterations, as the epididymis is vital for male fertility.

The histological results of G2 rabbits showed prominent changes in the epididymis as epididymal cell shrinkage, vacuolation and apoptosis with decreased number of spermatozoa and increased number of sloughed cells in lumens of $\mathrm{CE}$ ducts. Also, there was a thickening of epididymal LP and SMF around the epithelium of epididymal duct while the interstitium contained a thick loose connective tissue that reduced the luminal diameter. Moreover, IHC stained sections showed a strong cytoplasmic immunoexpression of caspase-3 in epithelial cells of $\mathrm{CE}$ ducts compared to $\mathrm{G} 1$ rabbits. These results were supported by Azari et al. who mentioned that there is cell apoptosis, decreased epithelial height and luminal diameter of epididymis with thickening of epididymal basement membrane and interstitial tissue in drug abusers which impaired epididymal metabolism and enhance epididymal cell atrophy [22].

Decreased epithelial thickness and cellular vacuolation in this study is explained by oxidative stress that increases generation of $\mathrm{O} 2$ free radicals as hydrogen peroxide in various organs. All of these factors impaired the physiological functions of male reproductive system [23]. Giri et al. mentioned that the epididymis protects sperms from hazards of excessive ROS and provides many antioxidants while, metabolism of spermatozoa increases oxidative stress. Moreover, the main mechanism of drug toxicity and destruction of epididymal mucosa is oxidative damage that enhances generation of free radicals and induces tissue necrosis [24]. Effects of ROS include multiple cell injuries as membranous lipid peroxidation, oxidative modification of proteins, membrane damage, DNA lesions and altered gene expression that induce cell degeneration, cytoplasmic vacuolation, changes of caspase-3 expression and apoptosis of epithelial cells [25]. 
Tramadol intake enhances apoptosis by releasing caspases (proforms of apoptosomes called death-driving cysteine proteases) or the mitochondrial apoptogenic factors such as cytochrome $\mathrm{C}$ into the cytoplasm that directly activate caspases to cleave a set of cellular proteins and finally cause apoptotic changes [26].

These effects were explained by Raffa who mentioned that the epididymis is a frequent target of phospholipidosis by metabolism of tramadol mainly in the liver through cytochrome P450 then conjugation with glucuronic acid and sulphate resulting in cytoplasmic vacuolation of epithelial cells and occlusion of CE ducts by sloughed debris [27]. While; Lamas et al. mentioned that decreased epithelial thickness, cell swelling, epididymal injury and reduced lumen proportion is related to the ultrastructural findings of disturbed cell adhesion and cytoplasmic lipid droplets that promoted cell swelling and cytoplasmic degeneration [4].

The histological and IHC results of G3 and G4 were nearly the same as the structure of G1. The PSCE lining the duct, LP and SMF were apparently normal as those of G1 rabbits however; a few degenerated PCs were seen with focal vacuolation in G4. CE duct sections showed a weak cytoplasmic immunoexpression of caspase-3 protein in $\mathrm{CE}$ epithelial cells. Moreover, all of these histological and IHC findings were supported by the morphometrical results and statistical analysis showed a significant increase in the epididymal apoptotic index \% of epithelial cells, area $\%$ of epididymal duct collagen fibers and area \% of caspase- 3 immuno-expression in epithelial cells of G2 compared to GI, G3 and G4.

These data are in compliance with the results of Kooti et al. who demonstrated that most of natural products like TQ contain large amounts of total polyphenol molecules that can prevent cellular damage due to drugs by reducing production of ROS and inhibiting lipid peroxidation [28]. Usage of TQ is a good option to protect the reproductive system from diseases through complex mechanisms and various actions. It had a strong antioxidant and cytoprotective properties on many organs against the oxidative stress and cellular oxidative damage that are induced by multiple free radical generating agents. Moreover, TQ modulates multiple factors as cell proliferation, migration or apoptosis, enhances the actions of antioxidant enzymes and inhibits cellular inflammatory responses [29]. TQ induction of antioxidant enzymes activity in cells of male genital tract is responsible for neutralizing the free radical induced oxidative damage as superoxide dismutase (SOD), catalase and glutathione reductase. SOD has a vital role in antioxidative reactions as a primary scavenging enzyme to catalyze $\mathrm{O} 2$ into $\mathrm{H} 2 \mathrm{O} 2$, which can be removed by catalase [30].

Furthermore, TQ alleviates the hazardous effects of tramadol as it reacts directly with ROS and clears up them from the testicular and epididymal cells. It exerts antiinflammatory properties by preventing the formation of inflammatory mediators, prostaglandins, leukotrienes, cytokines, inflammatory macrophages and neutrophils that create inflammation. Also, it inhibits production of 5- lipooxigenase and reduces TNF, Cox-2 and nitric oxide production [31].

\section{Conclusion}

The present study concludes that tramadol intake exerts hazardous effects on epididymal structure and should be avoided without medical prescription while, TQ is a promising therapeutic agent that can minimize tramadolinduced infertility. Therefore, TQ intake with tramadol therapy is advised to protect the epididymis and preserve male fertility.

\section{Acknowledgements}

The author gratefully acknowledges Qassim University/ KSA represented by the Deanship of Scientific Research on the material support for this research under the grant number (1339-CAMS1-2016-1-12-S) at the academic year 2016 AD / $1438 \mathrm{AH}$.

\section{Conflict of Interest}

The author declares that he has no competing interests.

\section{References}

[1] Carlsson C, Johansson AK, Alvan G, Bergman K, Kühler T. Are pharmaceuticals potent environmental pollutants?: Part I: Environmental risk assessments of selected active pharmaceutical ingredients. Science of the total environment. 2006; 364(1):67-87.

[2] Asghari A, Akbari G, Beigi M, Mortazavi P. Effects of Tramadol Administration on Sperm Characteristics on Testicular Ischemia-Reperfusion Injury in Rat. Crescent J. of Medical and Biological Sciences. 2016; 3(4):119-22.

[3] Bassols J, Kadar E, Briz M, Pinart E, Sancho S, Garcia-Gil N, Badia E, Pruneda A, Bussalleu E, Yeste M, Casas I. Effect of culture conditions on the abstention of boar epididymal epithelial cell monolayers. Animal reproduction science. 2006; 95(3-4):262-272.

[4] Lamas CD, Cuquetto-Leite L, do Nascimento da Silva E, Thomazini BF, Cordeiro GD, Predes FD, Gollücke AP, Dolder H. Grape juice concentrate alleviates epididymis and sperm damage in cadmium-intoxicated rats. International journal of experimental pathology. 2017; 98(2):86-99.

[5] De Souza AP, Schorr-Lenz AM, Lucca F, Bustamante-Filho IC. The epididymis and its role on sperm quality and male fertility. Animal Reproduction. 2017; 14:1234-44.

[6] Ahmed MA and Kurkar A. Effects of opioid (tramadol) treatment on testicular functions in adult male rats: The role of nitric oxide and oxidative stress. Clin. Exp. Pharmacol. Physiol. 2014; 41(4):317-325.

[7] Alkafafy M, Rashed R, Emara S, Nada M, Helal A. Histological and immunohistochemical studies on the epididymal duct in the dromedary camel (Camelus dromedarius). Anatomy \& cell biology. 2011; 44(4):284-294. 
[8] Omar NM. Nigella sativa oil alleviates ultrastructural alterations induced by tramadol in rat motor cerebral cortex. Journal of Microscopy and Ultrastructure. 2016; 4(2):76-84.

[9] Tewari SN, Salman MT, Thadani S, Singh S, Ahmad A. A study of pregabalin, tramadol, their combination and nigella sativa in neuropathic pain in rats. International Journal of Pharmaceutical Sciences and Research, 2015; 6(10):4406.

[10] Sandilands EA, Bateman DN. Opioids. Medicine, 2016; 44(3):187-189.

[11] Ragab IK, Mohamed HZ. Histological changes of the adult albino rat's entorhinal cortex under the effect of tramadol administration: Histological and morphometric study. Alexandria Journal of Medicine. 2016; 53:123-133.

[12] Mousa AM, Salem MM, El-Mahalaway AM. Effect of thymoquinone on cadmium-induced toxicity of Leydig cells in adult male albino rats: a histological, immuno-histochemical, and biochemical study. Egyptian Journal of Histology. 2015; 38(2):308-16.

[13] Abdel-Zaher AO, Abdel-Rahman MS, ELwasei FM. Protective effect of Nigella sativa oil against tramadol-induced tolerance and dependence in mice: role of nitric oxide and oxidative stress. Neurotoxicology. 2011; 32(6):725-33.

[14] Wang H, Liu YM, Qi ZM, Wang SY, Liu SX, Li X, Wang HJ, Xia XC. An overview on natural polysaccharides with antioxidant properties. Current medicinal chemistry. 2013; 20(23):2899-913.

[15] Elyazji NR, Abdel-Aziz I, Aldalou A, Shahwan O. The effects of tramadol hydrochloride administration on the hematological and biochemical profiles of domestic male rabbits. IUG Journal of Natural Studies. 2015; 21(2):51-65.

[16] Alkharfy KM, Ahmad A, Khan RM, Al-Shagha WM. Pharmacokinetic plasma behaviors of intravenous and oral bioavailability of thymoquinone in a rabbit model. European journal of drug metabolism and pharmacokinetics. 2015; 40(3):319-323.

[17] Bancroft JD, Layton C. The hematoxylins and eosin. In: Suvarna SK, Layton C, Bancroft JD. Editors. Bancroft's theory and practice of histological techniques, 8th ed., Philadelphia: Churchill Livingstone/Elsevier, 2018; (10):173214.

[18] El-Mahalaway AM, Salem MM, Mousa AM. The effect of potassium dichromate on convoluted tubules of the kidney of adult male albino rats and the possible protective role of ginseng: a histological and immunohistochemical study. Egyptian Journal of Histology. 2015; 38(2):157-67.

[19] Mousa AM. Effect of Pirfenidone on Bleomycin Induced Pulmonary Alveolar Fibrosis in Adult Male Rats (Histological, IHC, Morphometrical and Biochemical Study). International Journal of Clinical and Developmental Anatomy; 2016; $2(3): 17-23$.
[20] Tadros MG, Khalifa AE, Abdel-Naim AB, Arafa HM. Neuroprotective effect of taurine in 3-nitropropionic acidinduced experimental animal model of Huntington's disease phenotype. Pharmacology Biochemistry and Behavior. 2005; 82(3):574-82.

[21] Garcia PV, Barbieri MF, Perobelli JE, Consonni SR, de Fatima Paccola Mesquita S, de Grava Kempinas W, Pereira LAV. Morphometric-stereological and functional epididymal alterations and a decrease in fertility in rats treated with finasteride and after a 30-day posttreatment recovery period. Fertil Steril. 2012; 97:1444-1451.

[22] Azari O, Emadi L, Kheirandish R, Shafiei B. H, Esmaili Nejad MR, Faroghi F. The effects of long-term administration of tramadol on epididymal sperm quality and Testicular tissue in mice. Iranian Journal of Veterinary Surgery. 2014; 9(1):23-30.

[23] Žaja IŽ, Samardžija M, Vince S, Sluganović A, Strelec S, Šuran J, DelVechio I, Đuričić D, Ostović M, Valpotić H, Milinković-Tur S. Antioxidant protection and lipid peroxidation in TT and different parts of epididymis in boars. The riogenology. 2016; 86(9):2194-201.

[24] Giri A, Bharti VK, Angmo K, Kalia S, Kumar B. Fluoride induced oxidative stress, immune system and apoptosis in animals: a review. International Journal of Bioassays. 2016; 5(12):5174-8.

[25] Aboulhoda BE, Hassan SS. Effect of prenatal tramadol on postnatal cerebellar development: Role of oxidative stress. Journal of chemical neuroanatomy. 2018; 94:102-18.

[26] Mathew SJ, Manji HK, Charney DS. Novel drugs and therapeutic targets for severe mood disorders. Neuropsychopharmacology. 2008; 33(9):2080.

[27] Raffa RB. Basic pharmacology relevant to drug abuse assessment: tramadol as example. Journal of clinical pharmacy and therapeutics. 2008; 33(2):101-108.

[28] Kooti W, Hasanzadeh-Noohi Z, Sharafi-Ahvazi N, AsadiSamani M, Ashtary-Larky D. Phytochemistry, pharmacology, and therapeutic uses of black seed (Nigella sativa). Chinese journal of natural medicines. 2016; 14(10):732-745.

[29] Darakhshan S, Pour AB, Colagar AH, Sisakhtnezhad S. Thymoquinone and its therapeutic potentials. Pharmacological research. 2015; 95:138-158.

[30] Ismail $\mathrm{M}$, Al-Naqeep $\mathrm{G}$, Chan KW. Nigella sativa thymoquinone-rich fraction greatly improves plasma antioxidant capacity and expression of antioxidant genes in hypercholesterolemic rats. Free Radical Biology and Medicine. 2010; 48(5):664-672.

[31] Elkhateeb A, El Khishin I, Megahed O, Mazen F. Effect of Nigella sativa Linn oil on tramadol-induced hepato-and nephrotoxicity in adult male albino rats. Toxicology Reports. 2015; 2:512-519. 